\title{
Etiology of Pericardial Effusion and Outcomes Post Pericardiocentesis in the Western Region of Saudi Arabia: A Single-center Experience
}

\author{
Saad Albugami ${ }^{1}$, Faisal Al-Husayni ${ }^{2}$, Abdullah AlMalki ${ }^{2}$, Mohammed Dumyati ${ }^{3}$, Ysear Zakri ${ }^{2}$, Jamilah
} AlRahimi $^{1}$

1. Cardiology, King Faisal Cardiac Center, King Saud Bin Abdulaziz University for Health Sciences, King Abdullah International Research Center, Jeddah, SAU 2. Internal Medicine, National Guard Hospital, Jeddah, SAU 3. Internal Medicine, National Guard Health Affairs, King Abdulaziz Medical City, Jeddah, SAU

Corresponding author: Saad Albugami, sbugami@yahoo.com

\section{Abstract \\ Background}

Pericardial effusion is the accumulation of blood or excess fluid in the cavity between the heart and the pericardium sac. Pericardial effusion can be caused by several etiologies, including malignant and nonmalignant causes. Pericardiocentesis is the gold standard assessment method for pericardial effusion etiology. The aim of this study was to identify the long-term outcome of patients who presented with massive pericardial effusion and underwent pericardiocentesis at King Abdulaziz Medical City, Jeddah, a large tertiary hospital in the western part of Saudi Arabia.

\section{Methods}

This is a single-center retrospective cross-sectional study conducted at King Abdulaziz Medical City Jeddah, Saudi Arabia, between January 2013 to December 2018. Data were collected from patient's charts; the clinical and echocardiographic findings, alongside with pericardial fluid analysis, were collected. Procedure and patients outcomes were obtained and reported.

\section{Results}

Of the 107 patients with pericardial effusion, 39 patients had moderate to severe pericardial effusion requiring pericardiocentesis. The mean age was 52 years, and $56.4 \%$ were females. The most common chronic disease was hypertension and the presence of metastasis. The most common cause of pericardial effusion was a malignancy. A majority of patients had severe pericardial effusion. Many patients had tamponade (69.6\%). Patients with malignant pericardial effusion had a median survival of 54 days.

\section{Conclusion}

Received 01/07/2020 Review began 01/07/2020 Review ended 01/08/2020 Published 01/11/2020

\section{() Copyright 2020}

Albugami et al. This is an open access article distributed under the terms of the Creative Commons Attribution License CC-BY 3.0., which permits unrestricted use, distribution, and reproduction in any medium, provided the original author and source are credited.
Etiologies of pericardial effusion requiring drainage depend on the population studied. Patients with malignant effusions have worse outcomes than non-malignant effusion. Pericardiocentesis is required to ascertain the cause and risk-stratify patients.

\section{Categories: Cardiology}

Keywords: pericardiocentesis, pericardial diseases, pericardial effusion, cardiovascular diseases

\section{Introduction}

The normal pericardium is a double-layered sac that encircles the heart and roots of the large vessels. It is composed of two different layers; the outer one is the fibrous parietal pericardium, whereas, the inner one is the visceral pericardium [1]. The pericardium prevents the displacement of the heart and large vessels, prevents sudden dilatation of the heart, and the spread of infection or cancer from the pleura or lung as well as minimizes friction between the heart and surrounding structures [2]. The pericardial cavity is located between the parietal and visceral pericardium, and it is filled with 10-50 cc of fluid, which is ultrafiltrate of plasma and produced by the visceral pericardium. This fluid acts as a lubricant between the pericardium and the heart; however, blood accumulation and excess fluid in this cavity is called pericardial effusion [3-4]. The symptoms of pericardial effusion include cough, chest pain, dyspnea, and orthopnea [5]. Pericardial effusion develops in patients with diseases that affect the pericardium such as systemic disorders and pericarditis $[1,4]$. Pericardial effusion can be attributed to several etiologies, including malignant and non-malignant causes [6]. The known causes include neoplasia, infection, congestive heart failure, Iatrogenicity, radiation, trauma, connective tissue diseases, pericardial injury, and metabolic causes such as uremia and hypothyroidism; a substantial number of effusions are idiopathic [7-9]. 
effusion is symptomatic or significant [2]. It is also indicated when the effusion is accompanied by tamponade, or the cause of the effusion is uncertain [2]. Pericardiocentesis is the gold-standard method to ascertain the etiology of pericardial effusion [10]. This study was conducted to identify the etiology of pericardial effusion as well as the intermediate-term outcome of patients who underwent pericardiocentesis at King Abdulaziz Medical City in Jeddah, Saudi Arabia.

\section{Materials And Methods}

This research is an observational cross-sectional study conducted at King Abdulaziz Medical City, Jeddah. The study included all patients who underwent percutaneous pericardiocentesis between January 2013 till December 2018. Patients who had surgical drainage or were less than the age of 16 years were excluded. The data were collected from patients ' files, including sex, age, date of procedure, medical history, laboratory values, effusion size, fluid sample characteristics, clinical diagnosis, and date of death if available. Results of serological testing, if reported, cultures in peripheral blood, and pericardial fluid results were obtained. Effusion size was determined by reviewing pre-procedural echocardiogram reports. The small size was defined as $<10 \mathrm{~mm}$, medium $>10$ and $<20 \mathrm{~mm}$, and large $>20 \mathrm{~mm}$. Analyses of fluid characteristics, including macroscopic aspects, biochemistry, cytology, and microbiology, were collected.

\section{Statistical analysis}

Statistical analysis was conducted using STATA 12 software (StataCorp LP, TX). Continuous variables were presented as mean, standard deviation. Inter-group differences were compared using the t-test. Skewed numerical data were presented as median and average rank, and between-group differences were compared using the Mann-Whitney U test. Paired numerical data were compared using the paired t-test. Categorical variables were presented as number and percentage, and differences between groups were compared using the Pearson chi-squared test or Fisher's exact test. Ordinal data were compared using the chi-squared test for trend. Paired binary data were compared using the McNemar test, and paired ordinal data using the StuartMaxwell test of marginal homogeneity; p-values $<0.05$ were considered statistically significant.

\section{Results}

The hospital electronic system identified 107 patients with pericardial effusion. Among those, only 39 met the inclusion criteria. All patients had been diagnosed with pericardial effusion using echocardiography and were categorized into mild, moderate, and severe. The mean age was $52 \pm 19$ years old. Females represented more than half of patients 22 (56.4\%). Comorbid diseases were reported as follows; $28.2 \%$ had diabetes mellitus, 35.9\% had hypertension, while heart failure was seen in $18 \%$. Twenty percent had lung cancer; breast cancer was seen in $15.4 \%$ and lymphoma in $7.7 \%$. Metastasis was present among $35.9 \%$. Five patients (12.8\%) had autoimmune diseases, and one patient (2.6\%) was not known to have any medical illness. Patients' demographics and chronic diseases are shown in Table 1. 


\section{Cureus}

\begin{tabular}{|c|c|c|}
\hline Variable & Frequency & $\%$ \\
\hline Mean Age \pm SD (Median) & $52 \pm 19(54)$ & \\
\hline \multicolumn{3}{|l|}{ Gender } \\
\hline Male & 17 & 43.6 \\
\hline Female & 22 & 56.4 \\
\hline \multicolumn{3}{|l|}{ Chronic diseases } \\
\hline Diabetes mellitus & 11 & 28.2 \\
\hline Hypertension & 14 & 35.9 \\
\hline Chronic kidney disease & 8 & 20.5 \\
\hline Dyslipidemia & 1 & 2.6 \\
\hline Heart failure & 7 & 18 \\
\hline Lung cancer & 8 & 20.5 \\
\hline Breast cancer & 6 & 15.4 \\
\hline Lymphoma & 3 & 7.7 \\
\hline Multiple myeloma & 1 & 2.6 \\
\hline Prostate cancer & 1 & 2.6 \\
\hline Leukemia & 1 & 2.6 \\
\hline Metastasis & 14 & 35.9 \\
\hline Autoimmune diseases & 5 & 12.8 \\
\hline No medical illness & 1 & 2.6 \\
\hline
\end{tabular}

\section{TABLE 1: Demographics and chronic diseases of pericardial effusion patients}

The most common causes of pericardial effusion were malignancy (48.7\%), followed by infection, and uremia; both accounted for $15.4 \%$. (Table 2). Iatrogenic causes of pericardial effusion requiring pericardiocentesis were as follows: three cases (7.6\%) post-cardiac surgery and one case post arrhythmia ablation. Bacterial infection was the predominant cause of infected pericardial effusion (15.4\%).

\begin{tabular}{|l|l|l|}
\hline Cause & Frequency & $\%$ \\
\hline Idiopathic & 4 & 10.3 \\
\hline Malignancy & 19 & 48.7 \\
latrogenic & 4 & 10.3 \\
Infection & 6 & 15.4 \\
Heart failure & 3 & 7.7 \\
Uremia & 6 & 15.4 \\
Autoimmune & 2 & 5.1 \\
\hline
\end{tabular}

\section{TABLE 2: Etiology of pericardial effusion}




\section{Cureus}

of cardiac tamponade (Table 3).

\begin{tabular}{|l|l|l|}
\hline Severity & Frequency & $\%$ \\
\hline Mild & 0 & 0 \\
\hline Moderate & 7 & 18 \\
\hline Severe & 32 & 82 \\
Cardiac Tamponade & & 69.2 \\
\hline Yes & 27 & 30.8 \\
\hline No & 12 & \\
\hline
\end{tabular}

TABLE 3: Pericardial effusion severity for patients who underwent pericardiocentesis

The pericardial fluid appearance was bloody in 25 patients (64.1\%), while serous and serosanguinous appearances were $25.5 \%$ and $15.4 \%$, respectively. Eighteen percent showed malignant cytology. Pericardial fluid analysis is shown in Table 4.

\begin{tabular}{|l|l|l|}
\hline Appearance & Frequency & $\%$ \\
\hline Bloody & 25 & 64.1 \\
\hline Serous & 8 & 25.5 \\
Serosanguinous & 6 & 15.4 \\
Cytology & & 18 \\
Malignant & 7 & 82 \\
\hline Normal & 32 & 8 \\
\hline
\end{tabular}

TABLE 4: Pericardial fluid analysis

Following pericardiocentesis, medium-term outcomes are demonstrated in Table 5. Nineteen patients (50\%) died within two months. Re-accumulation was seen in $20.5 \%$ of the patients that required re-drainage. Procedure-related complications like arrhythmia, hematoma, and cardiac arrest were seen in 5.1\%, 5.1\%, and $2.6 \%$, respectively.

\begin{tabular}{|l|l|l|}
\hline Outcome & Frequency & $\%$ \\
\hline Re-accumulation & 8 & 20.5 \\
\hline Repeated pericardiocentesis & 3 & 7.7 \\
Arrhythmia & 2 & 5.1 \\
Hematoma & 2 & 5.1 \\
Arrest & 1 & 2.6 \\
Hypotension & 2 & 5.1 \\
\hline Death & 19 & 50 \\
\hline
\end{tabular}

TABLE 5: Outcome after pericardiocentesis 


\section{Cureus}

There was a significant association between malignant pericardial effusion and death (P-value 0.0001) (Table 6).

\begin{tabular}{|c|c|c|}
\hline Outcome & Chi2 & $P$ value \\
\hline Death & 15.2 & 0.0001 \\
\hline Re-accumulation & 0.76 & 0.38 \\
\hline Repeated pericardiocentesis & 0.41 & 0.51 \\
\hline Arrhythmia & 0.0014 & 0.97 \\
\hline Hematoma & 2 & 0.15 \\
\hline Arrest & 1 & 0.29 \\
\hline Hypotension & 2.2 & 0.136 \\
\hline
\end{tabular}

\section{TABLE 6: Correlation between malignancy and outcomes after pericardiocentesis}

The median survival of patients with pericardial effusion post drainage was reported to be 54 days, as shown in Figure 1.

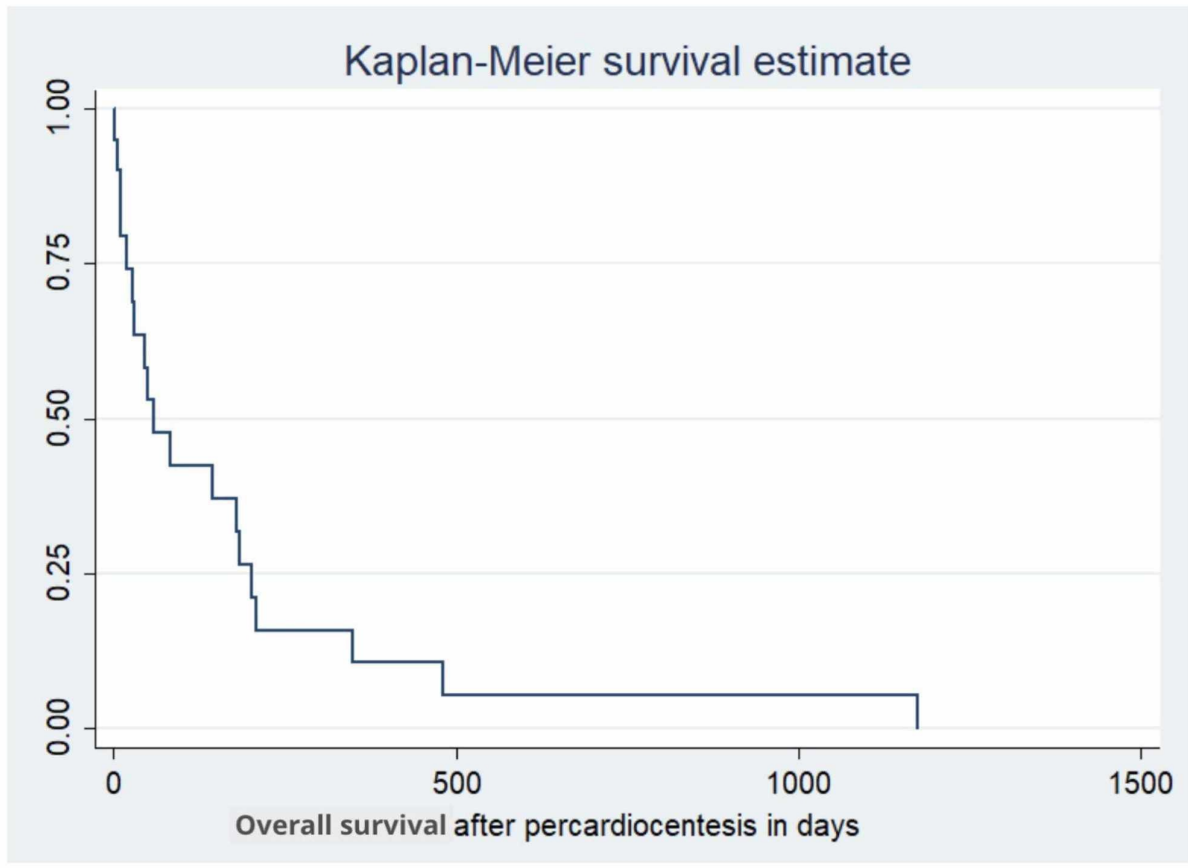

FIGURE 1: Kaplan-Meier curve showing overall survival of pateints following pericardiocentesis, with median of 54 days

\section{Discussion}

Uremic and tuberculous pericarditis were reported previously to be the most common causes of pericardial disease in the southern part of Saudi Arabia (Asir region), which may probably reflect the spectrum of diseases prevailing in that area [11]. Specific cause dominance of pericardial effusion depends on the population characteristics under study as well as the function of the healthcare facility they present to [12]. Historically, both malignancy and uremia were considered to be the most common causes of pericardial effusions [13]. Colombo et al. described 20 patients with pericardial effusion, $44 \%$ have presented with cardiac tamponade. Neoplastic (44\%), idiopathic (32\%), and uremia (20\%) were found to be the main reasons that cause cardiac tamponade [14]. Turak et al. described 104 patients with established moderate to severe pericardial effusion; idiopathic conditions were found to be the leading cause of pericardial effusions [15]. They also showed that malignancy, congestive heart failure, and tuberculosis were other primary etiologies 
that might lead to pericardial effusion. In another large study that consisted of 322 patients, 132 patients had moderate and 190 patients had severe pericardial effusion. Among them, the prevalence of cardiac tamponade was found to be $37 \%$. In that study, idiopathic (16\%), iatrogenic (16\%), and neoplastic conditions (13\%) were designated as common causes of pericardial effusion [16].

The current study was conducted on 39 patients from the western coast of Saudi Arabia. The most prevalent cause of pericardial effusion among these patients was malignancy (48.7\%). In contrast, infection and uremia were ranked second. This is probably because the hospital is a tertiary care center for oncology patients and has a large dialysis unit. Also, all infectious effusions were secondary to bacteria. Iatrogenic and idiopathic causes had the same prevalence of $10.3 \%$. There were $82 \%$ of patients with severe effusion; $69 \%$ of them had tamponade. The median overall survival was 54 days. It is not surprising that patients with malignant effusion had significantly worse survival. Our data is in agreement with what was reported by Strobbe et al. and El Haddad et al., both reported a worse survival among cancer patients [6,17].

Our report is the second one from Saudi Arabia, albeit from a different geographical area. It shows different results to what was reported previously, reflecting the divergent frequency of the underlying diseases from a different population.

\section{Limitations}

Our is a single-center, retrospective cross-sectional study. Certain variables, such as serological tests or clinical characteristics were not systematically recorded. Higher percentage of patients with cancer was included likely because the hospital has a large oncology tertiary care centre. Our patient population might not necessarily be representative of the region population due to selection bias. Besides, the total number was relatively small.

\section{Conclusions}

The majority of patients with large pericardial effusions necessitating drainage, in our study, are due to cancer. Malignant pericardial effusion carries a poor long-term prognosis. Extensive multicentre studies are required to compare populations, and ascertain causes relevant to each geographical area.

\section{Additional Information \\ Disclosures}

Human subjects: Consent was obtained by all participants in this study. King Abdullah International Medical Research Center issued approval RJ19/043/J. Animal subjects: All authors have confirmed that this study did not involve animal subjects or tissue. Conflicts of interest: In compliance with the ICMJE uniform disclosure form, all authors declare the following: Payment/services info: All authors have declared that no financial support was received from any organization for the submitted work. Financial relationships: All authors have declared that they have no financial relationships at present or within the previous three years with any organizations that might have an interest in the submitted work. Other relationships: All authors have declared that there are no other relationships or activities that could appear to have influenced the submitted work.

\section{References}

1. Little WC, Freeman GL: Pericardial disease. Circulation. 2006, 113:1622-32. 10.1161/CIRCULATIONAHA.105.561514

2. Jung HO: Pericardial effusion and pericardiocentesis: role of echocardiography. Korean Circ J. 2012, 42:725. 10.4070/kcj.2012.42.11.725

3. Munt BI, Moss RR, Grewal J: Pericardial disease. The Practice of Clinical Echocardiography, 4th Edition. Otto CM (ed): Saunders/Elservier, Philadelphia; 2011. 565-78.

4. Manning WJ: Pericardial disease. Cecil Medicine, 23rd Edition. Goldman L, Ausiello D (ed): Saunders/Elservier, Philadelphia; 2008. 548-52.

5. Singh D, Katlic MR: Malignant pericardial effusions. General Thoracic Surgery, 7th Edition. Shields TW, Locicero J, Reed CE, Feins RH (ed): Lippincott Williams \& Wilkins, Philadelphia; 2009. 885-90.

6. Strobbe A, Adriaenssens T, Bennett J, et al.: Etiology and long-term outcome of patients undergoing pericardiocentesis. 2017, 6:e007598. 10.1161/JAHA.117.007598

7. Imazio M, Mayosi BM, Brucato A, Adler Y: Pericardial effusion triage. Int J Cardiol. 2010, 145:403-4. 10.1016/j.ijcard.2010.04.031

8. Pugliatti P, Donato R, de Gregorio C, Patane S: A massive pericardial effusion in a cancer patient . Int J Cardiol. 2015, 181:138-40. 10.1016/j.ijcard.2014.11.141

9. Cubero GI, Rubin J, Martin M, Rondan J, Simarro E: Pericardial effusion: clinical and analytical parameters clues. Int J Cardiol. 2006, 108:404-5. 10.1016/j.ijcard.2005.03.037

10. Ertem A, Aytürk M, Duran M, Özkan S, Sunman H, Kılıç H, Yeter E: Etiology, diagnosis and management of severe pericardial effusion: a single center experience [Article in English, Turkish]. Dicle Tip Dergisi. 2014, 41:629-34. 10.5798/diclemedj.0921.2014.04.0489

11. Cheema MA, Ghalib MB, Shatoor AS, Suliman FA, Al-Hroub SS, Kardash M, Ahmed ME: Pattern of pericardial disease in the Asir Region of Saudi Arabia. Ann Saudi Med. 1999, 19:171-3. 10.5144/02564947.1999.171 


\section{Cureus}

12. Sagristà-Sauleda J, Mercé AS, Soler-Soler J: Diagnosis and management of pericardial effusion. World J Cardiol. 2011, 3:135-43. 10.4330/wjc.v3.i5.135

13. Osranek M, Bursi F, O’Leary PW, et al.: Hand carried ultrasound guided pericardiocentesis and thoracentesis. J Am Soc Echocardiogr. 2003, 16:480-84. 10.1016/s0894-7317(03)00080-4

14. Colombo A, Olson HG, Egan J, Gardin JM: Etiology and prognostic implications of a large pericardial effusion in men. Clin Cardiol. 1988, 11:389-394. 10.1002/clc.4960110606

15. Turak O, Gürel M, Çagli K, et al.: Pericardial effusion: etiology, diagnose and management [Article in English, Turkish]. Duzce Med J. 2012, 14:23-27.

16. Sagrista-Sauleda J, Merce J, Permanyer-Miralde G, Soler-Soler J: Clinical clues to the causes of large pericardial effusion. Am J Med. 2000, 109:95-101. 10.1016/s0002-9343(00)00459-9

17. El Haddad D, Iliescu C, Yusuf SW, William WN Jr, Khair TH, Song J, Mouhayar EN: Outcomes of cancer patients undergoing percutaneous pericardiocentesis for pericardial effusion. J Am Coll Cardiol. 2015, 66:1119-28. 10.1016/j.jacc.2015.06.1332 